Positions moyennes des étoiles de comparaison pour r888.0.

\begin{tabular}{|c|c|c|c|}
\hline * & $\alpha 1888.0$ & $\delta 1888.0$ & Autorité \\
\hline $\mathbf{I}$ & $23^{h} 5^{0^{m}} 26.86$ & $+32^{\circ} 5 x^{\prime} 53^{\prime \prime} 9$ & $W_{2} 23^{h} 1020$ \\
\hline 2 & $\begin{array}{lll}23 & 55 & 38.83\end{array}$ & +33254.9 & $w_{2} 23^{h} 145$ \\
\hline 3 & $\begin{array}{lll}23 & 5^{8} & 55.5^{\circ}\end{array}$ & $+333^{8} \quad 35.2$ & $w_{2} 23^{\mathrm{h}} \times 24^{0} .4^{1}$ \\
\hline 4 & $\begin{array}{lll}23 & 5^{6} & 3^{8} .4^{6}\end{array}$ & $\begin{array}{l}+34 \text { I3 } \\
2.9\end{array}$ & $W_{2} 23^{h} \times 169.70$ \\
\hline 5 & $0 \quad 351.07$ & $\begin{array}{lll}+34 & 41 & 4.4\end{array} \mid$ & $W_{2} 0^{\mathrm{h}} .22$ \\
\hline
\end{tabular}

\begin{tabular}{|c|c|c|c|}
\hline$*$ & $\alpha$ I 888.0 & $\delta$ I 888.0 & Autorité \\
\hline 6 & $0^{h} 9^{m} \quad 8: 54$ & $+35^{\circ} 4^{8} 22^{\prime \prime} 4$ & Lal. I 76 \\
\hline 7 & $0 \quad 153.20$ & $+3720 \quad 53 \cdot 3$ & ( Andr. $1 / 2\left(\mathrm{~N}_{7 y r}+9 \mathrm{yr}.\right)$ \\
\hline 8 & $02129.5^{2}$ & $1+39659.8$ & BB.VI $+38: 5 \mathrm{I}$ \\
\hline $\begin{array}{r}9 \\
0 \\
\end{array}$ & $\begin{array}{lll}0 & 23 & 6\end{array}$ & $\begin{array}{l}+394 \\
4\end{array}$ & DM. $+3^{8^{\circ}} 57$ \\
\hline 10 & 02427.26 & $1+3945 \quad 0.8$ & $W_{2} 0^{h} .570$ \\
\hline
\end{tabular}

Remarques.

Mai 13-17. Le noyau est allongé dans la direction du parallele; à la plus faible illumination du champ, quand les fils ne sont pas encore visibles, le noyau disparait; la queue mince, droite, très claire. Mai $x 6$ sa longueur fut $60^{\circ}$, la direction $=273^{\circ}$. Mai 17 les images très inquiètes.

Mai 20. Le noyau s'est concentré en un point et a la clarté d'une étoile de $8^{\mathrm{m}}$ ou même de $7.8^{\mathrm{m}}$, tandis que la queue parait être beaucoup plus faible qu'elle l'était; toutefois le ciel n'est pas pur. Le noyau est visible dans le champ bien éclairé.

Mai 22-23 le ciel fut couvert.

Mai 24. Le noyau est moins concentré, et en général l'aspect de la comète s'est changé. Le clair de la lune

empêche de mesurer la longueur de la queue principale qui est à peine visible; on voit beaucoup plus nettement les deux nouveaux rameaux qui se détachent de la tête dans la direction $\mathbf{N}$ et $\mathbf{S}$, se recourbant peu à peu à l'Ouest.

Mai 29. Le noyau est devenu si faible, qu' il est tres difficile de le voir dans le champ illuminé; le ciel n'est pas bien pur. Les deux rameaux latérals se sont élargis jusqu'à la queue principale.

Mai 31. Le noyeau se présente de nouveau allongé et délavé dans la direction de l'axe de la queue, dont la longueur fut $50^{\circ}$ dans la direction $272^{\circ}$. Depuis cette nuit le ciel est couvert.

Nicolaiew 18885 juin.

F. Kortazzi.

\title{
Observations of Comet 1888 I (Sawerthal)
}

made at the Melbourne Observatory.

\begin{tabular}{|c|c|c|c|c|c|c|c|c|c|c|c|c|c|c|c|c|c|c|c|}
\hline \multirow{2}{*}{$\begin{array}{c}888 \\
\text { Febr. } 23\end{array}$} & \multicolumn{3}{|c|}{ Melb. M. T. } & \multicolumn{2}{|r|}{$\Delta \alpha$} & \multicolumn{2}{|c|}{$\Delta \delta$} & \multicolumn{2}{|c|}{ Cp. Obs. } & \multicolumn{3}{|c|}{$\alpha$ app. } & \multirow{2}{*}{$\frac{\mid \log p .4}{9.84 \circ_{\mathrm{n}}}$} & \multicolumn{3}{|c|}{$\delta$ app. } & \multirow{2}{*}{$\frac{\log p .4}{0.265 \mathrm{n}}$} & \multirow{2}{*}{$\frac{\text { Red. ad l. app. }}{-2^{5.21}+8 . .1}$} & \multirow[t]{2}{*}{$*$} \\
\hline & & & $6^{\mathrm{m}} 39^{5} 8$ & & & & & 3 & $D$ & & & & & $-50^{\circ}$ & $0^{\circ}$ & & & & \\
\hline & & & & & & -10 & & 5 & B & & & & & & 58 & & & $-2.2 \mathrm{I}+8 . \mathrm{I}$ & \\
\hline 2 & & & $\begin{array}{ll}7 & 13.3\end{array}$ & - 0 & .59 & - & 5.9 & 6 & $\mathbf{W}$ & 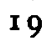 & 47 & 0.25 & & -48 & 34 & & & $-2.17+$ & \\
\hline 2 & & & $4 \quad 44.0$ & $\therefore 0$ & 2.7 & & 0.0 & 5 & $\mathrm{~B}$ & & 47 & & & -48 & 34 & & & $-2.17+$ & \\
\hline & & 22 & $5 \cdot 5$ & -3 & 23.0 & +5 & 4.1 & 5 & w & 1 & $5^{2}$ & & & -47 & 12 & & $7_{n}$ & -2.14 & \\
\hline & & 53 & 3 & -3 & & +7 & & 9 & B & & 52 & & & -47 & 10 & & $277 \mathrm{n}$ & -2.14 & \\
\hline & & & $\begin{array}{ll}6 & 32.8\end{array}$ & -2 & 3 & $+I I$ & 5.6 & 5 & B & 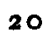 & 6 & & & -42 & 54 & & & $-I$ & \\
\hline & & 9 & 935.6 & -4 & & +4 & $24 \cdot 3$ & 5 & W & o & 6 & 3 & & -42 & 53 & & & -1 & \\
\hline \multirow[t]{12}{*}{ Mar. } & & 38 & 853.0 & -7 & & +6 & 24. & 3 & B & 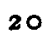 & 20 & & & $-3^{8}$ & 21 & & & -1 & \\
\hline & & I 7 & 717.6 & -7 & & +5 & 3.2 & 5 & W & o & 20 & 9. & & $-3^{8}$ & 323 & & & -1 & \\
\hline & & 51 & I 31.0 & -0 & 55.26 & +5 & 0.4 & 5 & $\mathbf{W}$ & o & 36 & II & & -32 & 14 & 46 & & $-\mathbf{I}$ & .7 \\
\hline & & 7 & 758.3 & -0 & & +13 & 53.0 & 5 & W & 1 & 43 & 3 & & -29 & 12 & 0.1 & & -1 & \\
\hline & & 28 & 817.6 & -0 & & +2 & & I 4 & $\mathbf{W}$ & 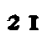 & 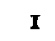 & & & $-2 \mathrm{I}$ & 35 & & & $-I$ & \\
\hline & & 5 & 5 & -0 & 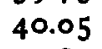 & -1 & 0.5 &. & W & $\mathbf{I}$ & 14 & & & -15 & 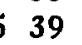 & & & $9-2$ & 10 \\
\hline & & 35 & 514.3 & -5 & 4 & -2 & 9.2 & 4 & w & $\mathbf{I}$ & 2 I & & & -12 & 47 & 49 & & $7-3.2$ & 1 \\
\hline & & 9 & 927.2 & -0 & $2.3^{2}$ & +5 & 31.1 & 10 & W & $\mathbf{I}$ & 27 & & & -10 & 3 & 5 & & $\begin{array}{lll}-1.21 & -3.7\end{array}$ & 12 \\
\hline & & 15 & $5 \quad 1 \times .6$ & -2 & 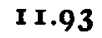 & -2 & & 6 & w & 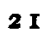 & 30 & & & - & 12 & .5 & & $18-$ & \\
\hline & & & 634. & -0 & 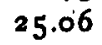 & +6 & 15 & 10 & B & & 34 & & & & 22 & & & -1 & \\
\hline & & & 124.7 & -5 & 5 & -9 & 28. & 5 & $\mathbf{W}$ & & 37 & & & & & & 0.7 & $6-$ & \\
\hline & & 714 & 421.9 & -2 & I 5.08 & -2 & 28.8 & I0 & W & I & 46 & & & -2 & 18 & 8.5 & $0.72 \mathrm{I}_{\mathrm{n}}$ & $-1.10-5.6$ & 10 \\
\hline \multirow[t]{4}{*}{ pril } & & $4^{x}$ & $x$ & -2 & & -2 & 50 & 6 & & & 2 & & & +3 & 30 & & & $3-7.0$ & \\
\hline & & ro & - 35 & -0 & . & 2 & & 5 & $\mathbf{w}$ & & 14 & & & & 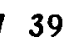 & & & & \\
\hline & & 41 & 143.6 & -6 & 4. & 170 & & 3 & B & 22 & 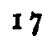 & & & +8 & 40 & & & $8-8.1$ & \\
\hline & & 729 & 92 & 1-I & 6 & 3 & 2 & & w & 22 & 23 & & & 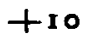 & 33 & & & .5 & \\
\hline
\end{tabular}

Observers: $\mathrm{W}=\mathrm{E}$. J. White, $\mathrm{B}=\mathrm{P}$. Baracchi. 
Mean Places of the Comparison Stars for I888.0.

\begin{tabular}{|c|c|c|c|c|c|c|}
\hline * & \multicolumn{3}{|c|}{$\alpha \mathbf{1 8 8 8 . 0}$} & \multicolumn{2}{|c|}{$\delta 1888.0$} & Authority \\
\hline $\mathbf{I}$ & I 9 & & 568 & $-49^{\circ} 4$ & $56: 5$ & Cord. ZC. 19.1672 \\
\hline 2 & I 9 & 47 & 7.01 & -483 & 19.0 & Cord. GC. 27227 \\
\hline 3 & 19 & 55 & 37.14 & -47 I & 38.9 & Cord. GC. 27423 \\
\hline 4 & 20 & 4 & 41.91 & -43 & 30.2 & $\begin{array}{l}1 / 2 \text { (Cord. GC. } 27622+\text { St. } \\
10820)\end{array}$ \\
\hline 5 & 20 & 10 & 59.19 & -425 & $44 \cdot I$ & Ll. 8396 (Cord. and St.) \\
\hline 6 & 20 & 27 & 37.71 & $-3^{8} \quad 2$ & I 8.4 & Ll. $84^{8} 7\left(\right.$ St. $\left.10_{9} 65\right)$ \\
\hline 7 & 20 & 37 & 7.91 & $-32 \quad 1$ & 49.1 & Ll. 8539 (Cord. and St.) \\
\hline 8 & 20 & 43 & $44 \cdot 5^{6}$ & -292 & 54.6 & $\mathrm{AOe}_{2} 20886$ (Cord.) \\
\hline 9 & 2 I & 2 & 8.59 & -213 & $35 \cdot 3$ & $x$ Capric. (Greenw. C. 1885 ) \\
\hline IO & 2 I & 15 & 18.62 & $\begin{array}{ll}-15 & 3\end{array}$ & 47.6 & Ll. 41445 (7yr. I 76I ) \\
\hline
\end{tabular}

Melbourne 1888 April 27.

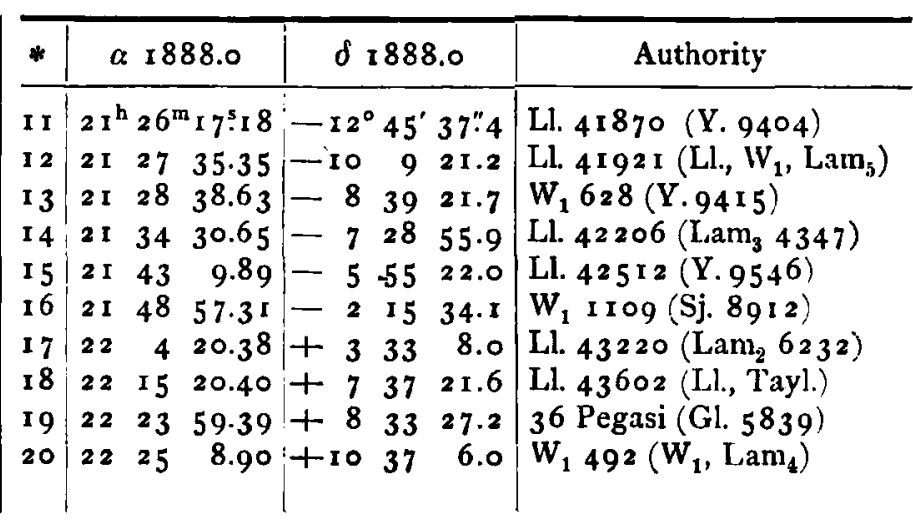

Rob. L. F. Ellery.

Ephemeride des Cometen 1888 I (Sawerthal).

(Fortsetzung z11 A. N. 2838.)

Die unerwartete plötzliche Zunahme der Helligkeit des Cometen Sawerthal lässt es als nicht unmöglich erscheinen, dass derselbe noch in den Herbstmonaten zu sehen sein wird. Auf jeden Fall wäre es wünschenswerth, den Ort des Cometen noch längere Zeit zu überwachen und dürfte deshalb die Fortsetzung der Ephemeride nicht überflüssig sein. Neue Elemente abzuleiten war einstweilen nicht nöthig, da die bisherige Rechnung mit den Beobachtungen ziemlich ubbereinstimmt, wie folgende Vergleichung einiger neuerer Positionen zeigt, deren Mittheilung ich zum Theil der Güte der Herren Beobachter selbst verdanke.

$$
\begin{aligned}
& \text { Mai I } 7 \text { Karlsruhe } \Delta a=-0.18 \Delta \delta=-3.5 \\
& \text { \. } 8 \text { Dresden }=+0.76=-7.1 \\
& \text { - } 18 \text { Padua }=+0.42=-27.6 \text { ? } \\
& \text {, } 24 \text { Karlsruhe }=+0.15=+2.3 \\
& 27 \text { Rom }=-0.03=-2.5 \\
& \text { * } 29 \text { Berlin }=+0.69=-13.9
\end{aligned}
$$

Die Unterschiede in diesen Abweichungen rühren theilweise von Unsicherheit der Vergleichsterne her, theilweise sind sie nicht ganz unbedeutenden Verschiedenheiten in der Auffassung des Cometenkernes durch die einzelnen Beobachter zuzuschreiben.*)

I $\mathbf{2}^{\text {h }}$ M. Z. Berlin.

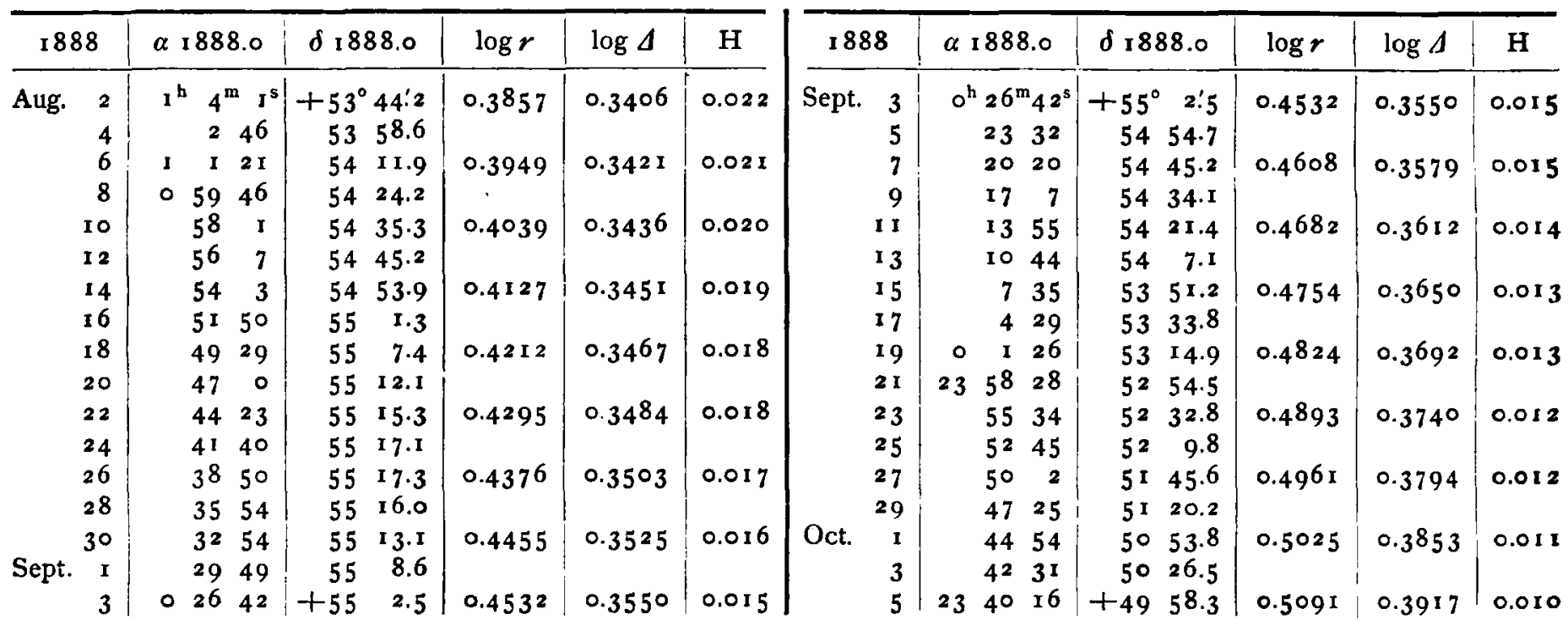

Die Helligkeit ist auf die vom 18 . Februar als Einheit bezogen.

Berlin 1888 Juni 6.

A. Berberich.

*) Mehrere Dresdener Beobachtungen aus der letzten Zeit geben für Anfang Juni die Correction der Ephemeride: +is und -o'.2. 